\title{
Study on the Intelligent Power Demand Response Technology for Smart Grid
}

\author{
Wenzhong Liu, Lu Wang, Jianwen Zhang, Jun Wang, Xiang Ge
}

State Grid Xinjiang Electric Power Research Institute, Urumqi, Xinjiang, 830011

Keywords: Intelligent Power Demand Response, Smart Grid, Key Technology

\begin{abstract}
Smart grid has become a major trend in the development of the world's power grid, in line with the inevitable requirements of social and economic development. The paper analyzes and summarizes the user demand response under smart grid conditions, and investigates the development status of domestic and international demand response. From the perspective of demand response concept, incentive mechanism, benefit evaluation, support platform technology, and application to wind power consumption, it is used at home and abroad. The scholars summarize the research results in related fields, analyze the typical cases, and point out the problems and related countermeasures of the current demand response, in order to provide reference for the development of smart electricity and demand response in China.
\end{abstract}

\section{Introduction}

Since 2002, the situation of continued power shortages has begun to emerge. In addition, the national strategy for smart grid development has proposed that demand-side management can participate in the grid in the form of demand response (DR) from the technical level and economic feasibility. "Two-way interaction", as an effective means to alleviate the contradiction of power supply and a strong support for the elimination of clean energy to achieve sustainable social development. In 2012, the Ministry of Finance and the National Development and Reform Commission approved four cities including Beijing, Tangshan, Suzhou and Foshan to become the first pilot cities for demand-side management. Different from the previous energy efficiency project-based pilots, this time will use the peak response of demand response transfer as an important task of the pilot work, marking that China's demand side management work has entered a new development stage. Traditional energy efficiency projects often produce continuous peak reduction and energy saving effects after equipment retrofit or renewal; however, the performance of demand response projects requires more attention to the user's participation in the project and various external factors in project execution. The impact of user responsiveness and willingness has greater uncertainty. As the leader of the DSM project, the government will have the problem of how to mobilize all sectors of society to achieve the lowest cost input of the DR project business operation model design; as the implementation body of the demand response project, the power companies at all levels often face the planning and design stage of the project. The following questions are as follows: 1 ) How can the DR project be designed to attract more users to participate in the project to increase the participation rate? 2) Will users respond positively to the incentives after participating in the DR project? Which factors affect their response level? 3) Facing the difference in characteristics Large and large number of participating users, how to assess their DR peaking potential and power saving potential, how to develop reasonable incentives to tap the above potentials, and to answer these questions, the key is the demand response characteristics of power users under different incentive levels The accurate grasp, in addition to the removal of incentives other than the impact factors (such as weather, whether there is demand response support technology, project peripheral policy environment), etc., make a reasonable judgment and analysis.

\section{Demand response and its influencing factors analysis}

The demand response is a concept proposed to play the role of the demand side in the competitive power market, thereby improving system reliability and market operation efficiency. It 
is demand side management in the competitive power market. Form of development. The demand response is firstly directed at the response behavior of price or incentive. Therefore, in a broad sense, the demand response refers to the user in the electricity market responding to the price signal or the incentive mechanism, and rationally adjusting the market participation behavior of the user's electricity consumption, and the user response. Electricity price or incentive signal, actively reduce the load during high electricity price period or when system reliability is threatened, which is of great significance for the safe and economic operation of the power system.

Direct load control refers to the way in which the direct load control mechanism turns off or controls the user's electrical equipment through the remote control device during peak hours of the system. Direct load control mainly has three kinds of load control strategies: 1) directly shutting down the user's power equipment; 2) turning on/off the loop control of the power equipment, such as using a 20min open/20min off control cycle within 40min; 3) adjusting The user-side temperature setting value or other parameter setting value is adjusted, that is, the effect of turning off the power-consuming device is realized by changing the setting value of the temperature or other parameters. The most commonly used direct load control items are remote control and regulation of electrical equipment such as air conditioners or water heaters, which are controlled by a digitally controlled receiver connected to the air conditioner's compressor and the water heater's internal immersion heater. It has been found through research that the next air conditioner can help reduce the load of $1 \mathrm{k} \mathrm{W}$, and a water heater can reduce the load by $0.6 \mathrm{k} \mathrm{W}$.

The interruptible load is a method of interrupting part of the power supply by the interruptible load implementing mechanism to the user during the peak period of the power grid according to the prior contract agreement between the supplier and the buyer. For users who do not have high reliability requirements for power consumption, part of the power consumption can be reduced or stopped to avoid grid spikes, and corresponding interrupt compensation can be obtained. The interruptible load is generally suitable for large industrial and commercial users, and is an ideal control method for grid peaks.

The demand side bidding bidding is an implementation mechanism for demand side resources to participate in the power market competition. It enables users to actively participate in market competition and obtain corresponding economic benefits in the form of bidding by changing their own electricity consumption mode, instead of simply The recipient of the price. Power supply companies and large users can directly participate in demand side bidding, while small decentralized users can participate in demand side bidding indirectly through third-party integrated load agencies.

The emergency demand response refers to a way for the user to interrupt the power demand in response to an emergency in an emergency situation and respond to the grid load adjustment request and the electricity price level. It combines historical data, price data, and short-term load forecasting to reduce peak loads and avoid spikes. However, due to the voluntary nature of emergency demand response projects, such projects are also unable to be a reliable load reduction resource.

Capacity market/auxiliary services are a form in which users provide reduced load as a system backup, replacing traditional generator sets or providing resources. In addition to the commitment to reduce the load when required, participants in the capacity market project must also demonstrate their ability to ensure the availability and sustainability of such reductions. For example, the NYRP SCRP program requires participants to have at least a reduction of $100 \mathrm{k} \mathrm{W}$ load, at least $4 \mathrm{~h}$, and an advance notice of $2 \mathrm{~h}$, with a capability test and audit for each review period. A load service agency that is eligible to participate in the project can obtain capacity payments or offset the capacity requirements of the power generation installed capacity market.

\section{Demand response mechanism and key technology research}

The demand response refers to the behavior of the power user responding to the price signal or incentive mechanism to change the behavior of the inherent custom power mode. The important part of implementing the demand response project is the response behavior of the power users to the incentives of the power company, and the change of the load characteristics caused by the power 
users adjusting their own power consumption mode. The way and intensity of this response behavior depends on the response characteristics of the users themselves. . Demand response measures can be divided into two types according to different response modes of users: price-based demand response (PBDR) and incentive-based demand response (PBDR). In the price-based demand response project, the price has the greatest influence on the user's power consumption behavior. The demand price elasticity is generally used to quantitatively characterize the impact of the electricity price change on the user's response behavior characteristics. Load price elasticity is often used to reflect the sensitivity of electricity consumption demand to electricity price changes. Alternative elasticity is also used to measure the change in the proportion of electricity used during the peak period of electricity price change and electricity consumption during the valley period. In the case of a small amount of data in the project, arc elasticity can be used to describe the price elasticity of demand. There are also literatures that use multi-agent approaches and power consumer psychology models to simulate user response to price. In the stimulus-based demand response project, the response characteristics of interruptible load, direct load control, etc. are generally characterized by characteristics such as response volume, response speed, response duration, response frequency, response interval time, responsiveness, and response notification time. Modeling. The idea of regression is also used to simulate the response of power users to different electricity prices and excitation signals under specific demand response items. When analyzing the user response characteristics, clustering the power users according to the demand response characteristics can simplify the cumbersome problem analysis and classify the user's demand response characteristics. With the rapid development of the smart grid, advanced communication, control and other demand response support technologies have enhanced the user's responsiveness. Demand response automation increases the flexibility and efficiency of demand response, making grid and user engagement easier. Lawrence Berkeley National Laboratory has developed OpenADR, an open automated demand response communication specification for demand response automation.

In the demand response project, according to different participants and different demand response projects, the decision variables, decision objectives and constraints in the optimization decision are also different. When the power company participates in the power generation dispatch optimization decision based on the incentive demand response, the decision variable is the load reduction and reduction time of each demand response user or load aggregator, or the direct load control control strategy. The objective function is generally the lowest power generation cost, minimized network loss, minimized incentive compensation for demand response projects, maximizes its own revenue, minimizes congestion compensation, and minimizes carbon emissions. When the price-based demand response is involved in the optimization decision, the decision variable is the electricity price policy that can meet the peak-filling requirements. The optimization target is generally the maximum peak load of the daily load curve, the peak-to-valley difference of the daily load curve, and the user. Maximize satisfaction and so on.

The demand response participates in the operation of the electricity market through load adjustment, which improves the efficiency of the use of systems and resources, and plays an important strategic role in the power industry and economic development as well as environmental protection. The benefits of the demand response project include the direct benefits of user-side electricity cost savings, economic compensation, reduction of grid-side electricity purchase costs, and reduction of reliability costs through the implementation of the project, including public benefits and external benefits. External benefits such as natural resource benefits. However, to accurately assess the benefits of demand response to different entities in the power system, these benefits need to be quantified. Quantitative analysis of the benefits of demand response is of great significance for grasping the development potential of demand response and promoting the implementation of demand response projects. The multi-dimensional analysis of the comprehensive benefits of demand response can be carried out from the main body, time and project, and the deconstruction idea is used to realize the quantification of benefit evaluation.

In the demand response project, the design of the incentive mechanism is the key. In the incentive-based demand response project, the user's participation in the demand response can be 
compensated in two ways: one method is similar to the independent system operator ISO (independent system operator), the power exchange center PX (power exchange) and other institutions to the user The blackout price is evaluated; the other method is to declare the interruptable load capacity and its corresponding power shortage cost by the user [55]. In the latter case, the user has a tendency to strategically report the cost of power shortage. By designing an incentive mechanism that is compatible with incentives, the user can obtain the maximum profit when reporting the real type, and encourage the user to report the true power shortage cost. There are also literatures based on credit integral incentive theory, calculating consumption, reward and punishment points, and using excess points to exchange electricity bills or avoiding electricity.

\section{Conclusion}

With the vigorous development of China's smart grid, the requirements for grid reliability and energy efficiency are getting higher and higher. Intelligent power consumption, which is an inevitable development trend of electric energy use, is an important part of smart grid. Its core feature is flexible two-way interaction between power grid and users, and demand response is one of the most important implementation methods of energy interaction, through price or Incentives and other methods guide the user to change the power load to participate in the peaking of the power grid, and actively participate in optimizing the power consumption mode by the user to increase the role of the demand side in the power market. Aiming at this purpose, this paper studies and analyzes the formulation and implementation of demand response strategy and user response behavior in the context of the development of intelligent power.

\section{References}

[1] Gao Ciwei, Liang Tiantian, Li Huixing, et al. Development and application of open automateddemand response [J]. Power System Technology, 2013, 37(3): 692-698

[2] Guo Lianzhe, Tan Zhongfu, Li Xiaojun. Demand response based model and method for optimal design of time-of-useelectricity price [J]. Power System Technology, 2006, 30 (5): 25-28.

[3] Ng K H, Gerald B S. Direct load control-A profit-based load management using linear programming [J]. IEEE Transactions on Power Systems, 1998, 13(2): 688-694.

[4] Liu Xiaocong, Wang Beibei, Li Yang, et al. Day-ahead generation scheduling model considering demand side interaction under smart grid paradigm[J]. Proceedings of the CSEE, 2013, 33(1): 30-38.

[5] Li Yuzeng, Liu Chang, Zhang Shaohua, et al. Multi-period dispatch of interruptible loads by intelligent optimization algorithms [J]. Journal of Electric Power Science and Technology, 2009, 24(4): 34-38. 